\title{
EDUCACIÓN VÍA STREAMING: UNA ALTERNATIVA PARA LA SOCIALIZACIÓN DE INVESTIGACIONES EN TIEMPOS DE LA PANDEMIA DE COVID -19
}

\section{EDUCATION VIA STREAMING: AN ALTERNATIVE FOR THE SOCIALIZATION OF RESEARCH IN TIMES OF THE COVID -19 PANDEMIC}

\author{
Jéssica Pamela Torres Lescano ${ }^{1}$ \\ Rómulo Arteño Ramos, \\ Profesor investigador ${ }^{2}$ \\ Hernán Paúl Ortiz Coloma ${ }^{3}$ \\ Mery Elizabeth Zabala Machado 4
}

Ecuador

\footnotetext{
1 Jéssica Pamela Torres Lescano, Doctoranda en Historia de los Andes, Facultad Latinoamericana de Ciencias Sociales FLACSO, Ecuador, correo jesspamela.26@ gmail.com ORCID: https://orcid.org/0000-0001-9102-0012 2 Rómulo Arteño Ramos Profesor investigador, Magíster en Geografía, Universidad Nacional de Chimborazo, Riobamba- Ecuador grupo de investigación Puruhá, correo rramos@unach.edu.ec ORCID: https://orcid. org/0000-0001-9606-6546 3

3 Hernán Paúl Ortiz Coloma, Docente Pontificia Universidad Católica del Ecuador, PUCE Sede Ambato, correo hortiz@pucesa.edu.ec ORCID: https://orcid.org/0000-00023374-6015

$4 \quad$ Mery Elizabeth Zabala Machado Profesora investigadora Magister en currículo carrera de Ciencias Sociales Universidad Nacional de Chimborazo, Riobamba -Ecuador correo mzabala@unach.edu.ec ORCID https://orcid.org/ 0000-0002-7559-278X
}

\section{RESUMEN}

La investigación exploró la utilidad de la educación vía Streaming para la divulgación de artículos científicos en el contexto de pandemia causada por la COVID -19 en el año 2020 mediante la medición y reflexión de los alcances y límites de la publicación digital. La estrategia tecnológica se produjo en el marco de la celebración del Bicentenario de la Independencia de los países de América Latina. Los estados -nación adaptaron los programas de rememoración al nuevo contexto 
de salud mundial buscando alternativas que no generen aglomeraciones en los espacios tradicionales de celebración. Las iniciativas provinieron de organismos públicos y privados. En el caso del cantón Ambato en Ecuador la decisión fue impulsada de la página de difusión histórica y cultural "Ambato ayer y hoy" con el evento denominado "Jornadas Académicas de Historia por el Bicentenario de Ambato". La investigación socializada en las Jornadas que se va rastrear aquí es la titulada "Tiempo de Fiesta: Celebraciones cívicas del siglo XX" por considerarse una de las más cercanas a la temática del Bicentenario. Finalmente, consideramos que la educación vía streaming como lo señalaron los datos es una de las mejores opciones que conjuga el aprendizaje en doble vía sincrónico y diacrónico con la interacción del espectador y los autores de la investigación.

\section{PALABRAS CLAVE}

Educación, streaming, socialización, historia, COVID 19

\section{ABSTRACT}

The research explored the usefulness of education via Streaming for the dissemination of scientific articles in the context of the pandemic caused by COVID-19 in 2020 by measuring and reflecting on the scope and limits of digital publication. The technological strategy was produced within the framework of the celebration of the Bicentennial of the Independence of the Latin American countries. The nation-states adapted the remembrance programs to the new context of world health, seeking alternatives that do not generate crowds in the traditional spaces of celebration. The initiatives came from public and private organizations. In the case of the Ambato canton in Ecuador, the decision was prompted by the historical and cultural dissemination page "Ambato yesterday and today" with the event called "Academic Days of History for the Bicentennial of Ambato." The research socialized in the Conference that will be traced here is the one entitled "Fiesta Time: Civic Celebrations of the 20th Century" because it is considered one of the closest to the theme of the Bicentennial. Finally, we consider that education via streaming.

As the data indicated, it is one of the best options that combines synchronous and diachronic dual-track learning with the interaction of the viewer and the authors of the research.

\section{KEYWORDS}

Education, streaming, socialization, history, COVID 19

\section{INTRODUCCIÓN}

La organización de las fiestas cívicas y las conmemoraciones centenarias eran eventos que modificaban el transcurrir cotidiano de las autoridades y actores sociales locales. En muchos casos, acercarse a la fecha oficial de las celebraciones comprometía a los Concejos Cantonales a preparar con anticipación la elaboración de una agenda local o nacional. Estas actividades promovidas por las autoridades locales podían estar auspiciadas o respaldadas por redes institucionales provenientes de círculos intelectuales y económicos.

Durante inicios del siglo XX las agendas de las celebraciones cívicas incluyeron la inauguración de infraestructura local y actividades educativas. En Ambato, por ejemplo, para la celebración del Centenario del primer grito de independencia se acordó en el Concejo Cantonal la entrega del parque y la estatua del ilustre escritor Juan Montalvo, reivindicar la memoria del poeta Juan León Mera como autor del himno nacional, el establecimiento de 
una fuente de energía eléctrica para la ciudad, la promoción de un concurso literario, para premiar el mejor estudio biográfico de Montalvo, la inauguración de la Escuela Normal de Agronomía Luis A. Martínez (Nicola 2019, 146, 147).

Desde esta perspectiva, la investigación surgió como iniciativa por el acercamiento de la conmemoración del bicentenario de independencia de Ambato el 12 de noviembre del 2020 en un contexto de pandemia que conllevó a modificar las agendas y los espacios tradicionales de celebración. Los 200 años de independencia han motivado la construcción de agendas independientes $\mathrm{o}$ auspiciadas por varias instituciones políticas, académicas y culturales que se adaptaron a la nueva normalidad de forma digital.

En lo local, se emprendieron iniciativas desde la entidad encargada tradicionalmente del festejo. Si en la conmemoración del primer grito de independencia o el centenario de independencia la entidad encargada del programa fue el Concejo Cantonal, en la conmemoración del bicentenario lo fue el Gobierno Autónomo Descentralizado de Ambato. El proyecto "Ambato en el Bicentenario" incluyó la elaboración de artículos de difusión, webinars y podcast. La agenda también provino de entidades educativas locales con el lanzamiento y presentación de libros, conferencias.

Si bien, en los programas mencionados provienen de entidades públicas nos preguntamos sobre las iniciativas de entidades privadas dedicadas a la difusión cultural como la página virtual de divulgación de historia local llamada "Ambato Ayer y hoy" en colaboración con entidades locales y nacionales como la Academia Nacional de Historia del Ecuador Capítulo Ambato y la Casa de Montalvo. ¿De qué manera la educación vía streaming en plataformas digitales privadas representan una alternativa efectiva para la divulgación de las investigaciones en contextos de celebraciones cívicas locales?

La página virtual de divulgación de historia local llamada "Ambato Ayer y hoy" empezó en la plataforma digital Facebook el año 2015 por la iniciativa del Doctor Hernán Ortiz Miranda. Las primeras publicaciones se trataron de noticias locales y fotografías que con el pasar de los años se fueron sumando y clasificándolas en secciones "Ambato antiguo", "Reinas deAmbato", "Atrios de la catedral", "Deportes", "Personajes históricos", "Personajes contemporáneos" y "documentos históricos". En la actualidad la página cuenta con 67058 seguidores y 62500 "me gusta" y es administrada por el Doctor Paul Ortiz Coloma. Datos que nos sirven para afirmar que la pagina se catapultó como una de las mas importantes a nivel local.

La organización de las agendas desde las plataformas privadas nos hace pensar en la inclusión de discusiones históricas, culturales como un deber social. Una conmemoración centenaria es el elemento que activa este deber cívico de las instituciones. Además, se establecen lineamientos de qué recordar, para qué recordar, a quienes recordar y quien es la voz oficial que establece ese recuerdo. En este contexto, rastrearemos el proceso organizativo de las "Jornadas Académicas de Historia por el Bicentenario de Ambato" en el caso particular de la ponencia "Tiempo de Fiesta: Celebraciones cívicas del siglo XX" y la elección de la metodología streaming para su socialización.

\section{METODOLOGÍA}

El Streaming es una técnica de transmisión de información multimedia con una ventaja de la posibilidad de reproducción del archivo conforme se va recibiendo, sin la necesidad 
de descargar el archivo completo. Es decir, la posibilidad de la recepción en tiempo real sin la ocupación de espacio en el dispositivo (Alvarez). En el contexto de pandemia la técnica streaming ha permitido suplir de alguna manera las conferencias, charlas, talleres y otros eventos académicos.

La presente investigación rastreó la utilidad de la técnica streaming de la página de Facebook "Ambato ayer y hoy" para el desarrollo de una actividad académica en conmemoración del Bicentenario de Independencia de la localidad. La intención es identificar el proceso de organización, desarrollo y ejecución de las jornadas académicas para el caso de la ponencia sobre las celebraciones cívicas como una posible alternativa de difusión cultural para posteriores festejos locales.

La investigación es de carácter mixto. Es cuantitativa en la medida en que integra los cuadros estadísticos que nos proporcionar la página Ambato ayer y hoy. Los porcentajes serán empleados en el proceso de logística de las jornadas: la identificación de la población objetiva y la evaluación de los resultados como la cantidad de reproducciones e interacciones.

Es una investigación cualitativa porque tomando en consideración la discusión bibliográfica sobre la técnica de transmisión streaming y la ponencia sobre las celebraciones cívicas en el siglo $X X$ que se incluye en las Jornadas por el Bicentenario abordamos las ventajas de la socialización histórica y cultural. El diálogo entre los datos cuantitativos y los debates teóricos es eje trasversal del artículo.
Los instrumentos para la investigación mixta son dos. Primero, se desarrolla una hoja de ruta para las jornadas que incluyen la logística, organización, selección de temáticas, detalles de la presentación. Y, la tabulación de datos estadísticos que nos proporciona la página "Ambato ayer y hoy" para evaluar la transmisión en vivo y las interacciones lo que permitiría calificar como útil el uso de la técnica streaming.

Para considerar el público objetivo o público meta fue necesario explorar las estadísticas de la página Ambato ayer y hoy. De acuerdo, el especialista en Marketing moderno, Philip Kotler (2001) un público es cualquier grupo que tiene un interés o impacto real o potencial en la capacidad de una compañía para lograr sus objetivos (78). De ahí que, los datos sobre el público permitieron elegir la técnica streaming como la mejor forma para socializar las jornadas académicas.

Los datos que se muestran a continuación son el número de personas que hay visto las publicaciones al menos por una vez, agrupadas por edad y género considerando los datos de los usuarios en sus perfiles de Facebook. La franja de mayor circulación de la página "Ambato ayer y hoy" es de un público objetivo entre los 18 a 44 años. De los cuales, $42 \%$ son mujeres y un $47 \%$ son hombres. 
Tabla 1. Población objetiva por género

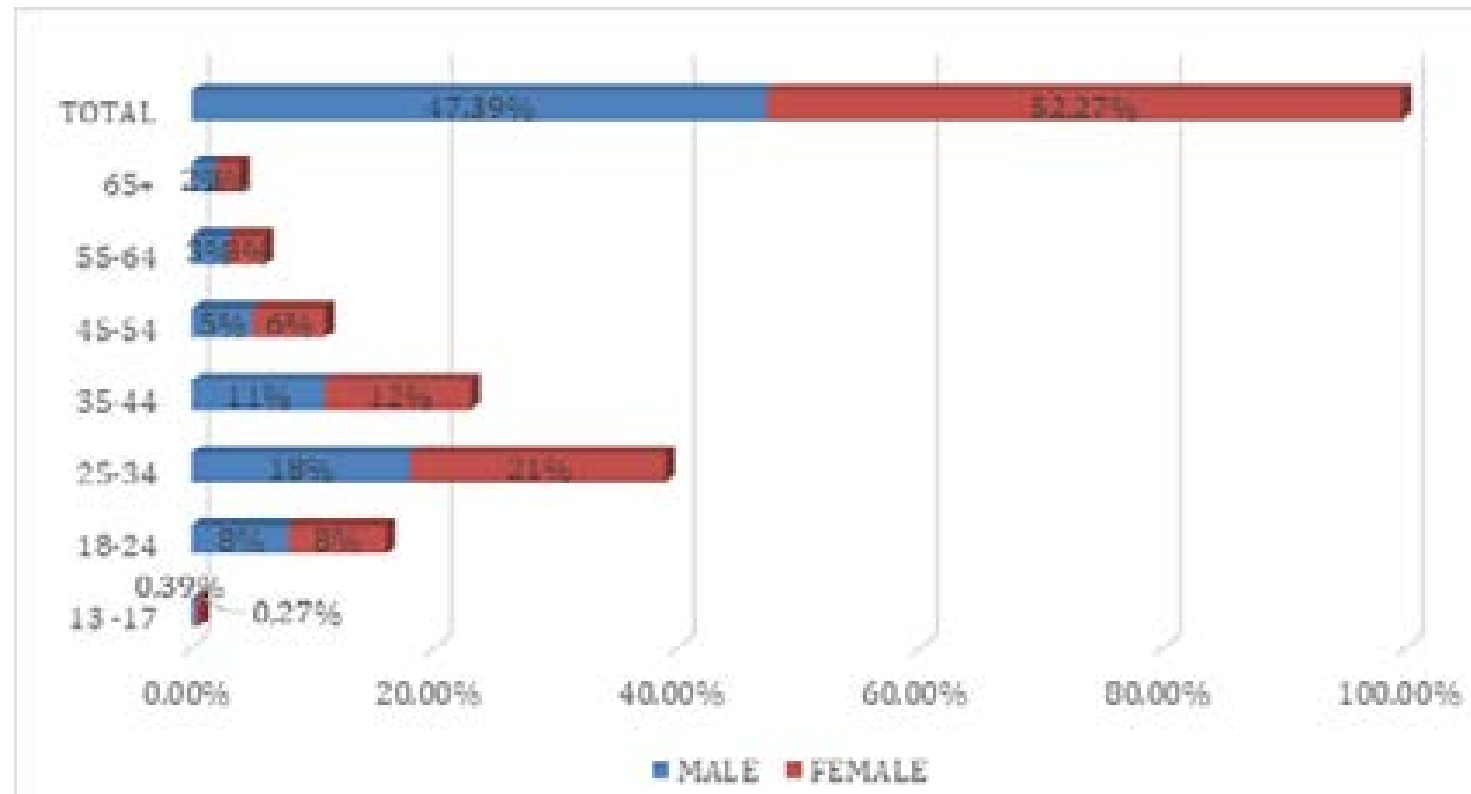

Fuente: Página de Facebook Ambato ayer y hoy

Elaborado por: Jéssica Pamela Torres Lescano, Rómulo Arteño Ramos, Hernán Paul Ortiz Coloma, Mery Elizabeth Zabala Machado

La duración del estudio fue de 9 meses, que comprendió dos fases. La primera de planificación, ejecución de las jornadas. Es decir, desde inicios de agosto de 2020 hasta noviembre de 2020. Esta fase fue importante para establecer una especie de metodología de elaboración de eventos académicos usando la técnica streaming. $Y$ la segunda fase de evaluación que la realizamos el mes de abril de 2021 que resulta útil para determinar el alcance a 5 meses de culminado el evento.

\section{RESULTADOS}

La elaboración de proyectos requiere de pautas de ruta vistas más como guías metodológicas y no como recetarios que se deben seguir al pie de la letra. Esta parte del artículo pretende explicar una serie de lineamientos para desarrollar las jornadas académicas de historia por el bicentenario hasta culminar con su ejecución y evaluación relacionándola con la educación via streaming. La identificación de la metodología permitió precisar la actividad que se quiere realizar y seleccionar los recursos. Esta vez nos acercaremos a los cursos de acción que nos conduzcan a los productos y resultados y finalmente, a establecer criterios de evaluación para valorar lo realizado (Ander Egg y Aguilar Idáñez 2005, 13).

El diagnóstico, -la primera fase- determinó las primeras pautas para la elaboración del proyecto de difusión cultural. Entre los datos relevantes que se determinaron fueron el público destinatario del proyecto que va entre los $18 \mathrm{y}$ 42 años de edad, los objetivos de las jornadas académicas de historia para la socialización de investigaciones por el bicentenario y las estrategias de divulgación determinándose la vía de transmisión por streaming como la más adecuada.

La segunda fase del proyecto es la producción del proyecto. El plan de comunicación de las jornadas se desarrolla en esta fase. La finalidad es difundir la propuesta para dirigirse al público destinatario. No se trata solamente 
de realizar publicaciones en redes sociales del evento. Sino de saber cómo y qué comunicar. Es recomendable que este tema se lo haga con expertos profesionales en comunicación, marketing y diseño (Cerezuela 2013, 16).

Para esta sección es importante elaborar un plan de comunicación en la página de Facebook de Ambato ayer y hoy. En el plan de comunicación se recomienda identifica el público objetivo, desarrollar un concepto para la campana y evaluar y medir el impacto de la comunicación (Eidus - Concello da Coruña 2020, 3). De la mano de la diseñadora y del comunicador social se diseña como plan de comunicación la elaboración de afiches publicitarios. Un afiche general de las jornadas académicas que incluya a todos los conferencistas, la fecha y la hora. $Y$ otro afiche de cada investigador con una fotografía que lo identifique, la fecha, hora y el tema de la presentación.

\section{Imagen 1}

\section{Diseño del afiche: Jornadas Académicas por el Bicentenario}

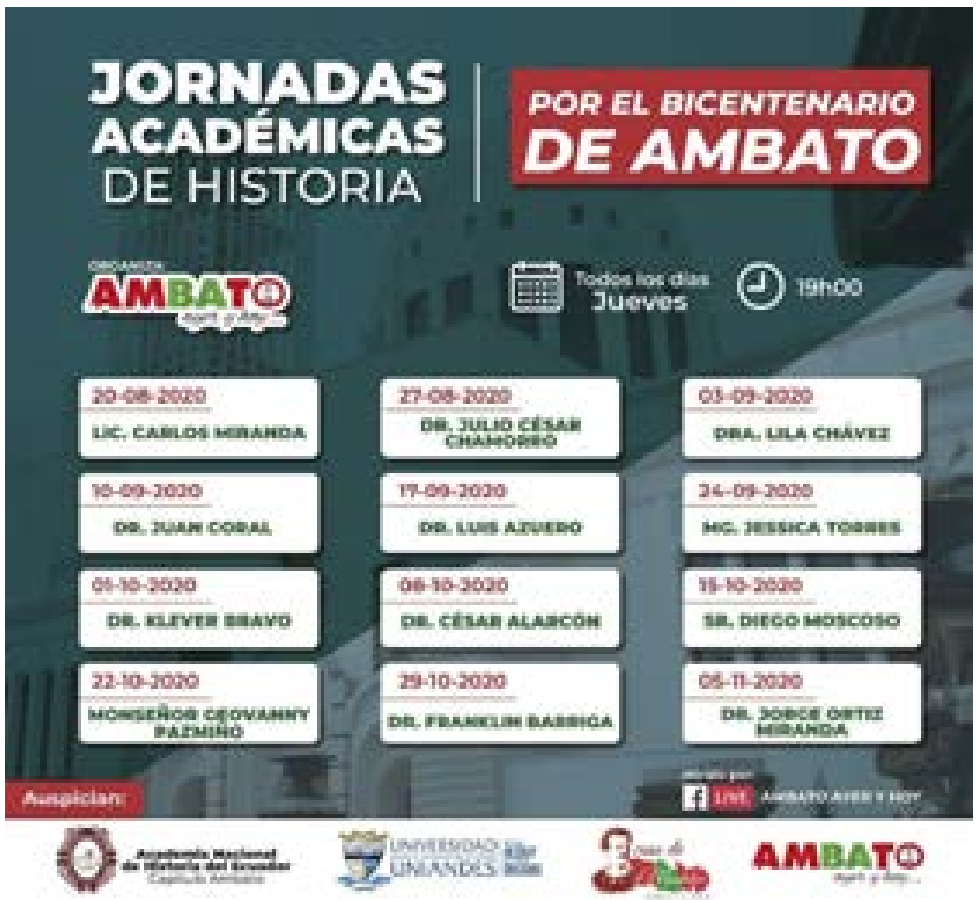

Fuente: Página de Facebook "Ambato ayer y hoy"

Elaborado por: Diseñadora "Ambato ayer y hoy" 
La finalidad de esta etapa es concretar en planes de acción, ejecutables y acotados en el tiempo, las estrategias planteadas con anterioridad (Tur-Viñes y Monserrat-Gauchi 2014). La elaboración de los afiches es de utilidad para promover una comunicación estratégica que permita trabajar con objetivos, diseñar un cronograma. En un contexto de imprevisibilidad, la comunicación estratégica adquiere el rigor necesario, recurrentemente demandado por todos los agentes del mercado (Tur-Viñes y Monserrat-Gauchi 2014).

\section{Imagen 2}

Diseño del afiche: Ponencia "Tiempo de Fiesta: Celebraciones Cívicas en Ambato del siglo XX"

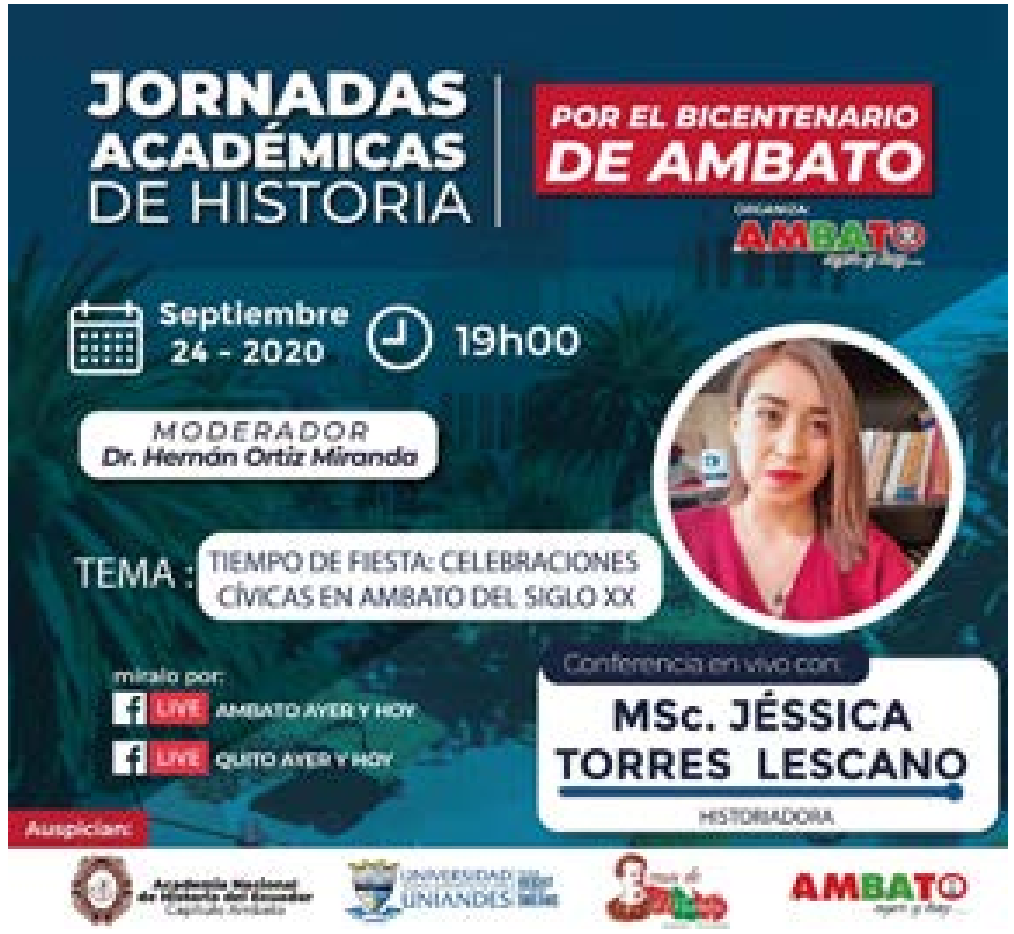

Fuente: Página de Facebook "Ambato ayer y hoy"

Elaborado por: Diseñadora "Ambato ayer y hoy"

Uno de los aspectos importantes del afiche es incluir la plataforma por las que fueron transmitidas las Jornadas Académicas. La plataforma de Facebook "Ambato ayer y hoy" y "Quito ayer y hoy" fueron los espacios para compartir las investigaciones históricas y ampliar el debate. Como estrategia se incluyó el logo de la plataforma "Facebook live" para que el público conozca la posibilidad de interactuar los investigadores y realizar preguntas 0 comentarios que aporten al debate.
Desde esta perspectiva, es necesario considerar que la educación online no consiste únicamente en cargar y descargar archivos de una plataforma virtual, radica en la búsqueda de estrategias didácticas, las cuales deben ser de fácil comprensión y de este modo los estudiantes afiancen los contenidos (Cáceres Piñaloza $2020,1)$. De manera que, la intención de las Jornadas Académicas es generar espacios de convivencia y de afectividad entre los investigadores y el público. De ahí, la importancia 
de entablar canales de comunicación al final de las conferencias.

En la ejecución del proyecto fue importante elaborar una hoja de ruta para la intervención de cada participante. La planificación de cada actividad debe ser vista como una herramienta que sirva para la consecución de los objetivos. Se trata de la parte más tangible del proyecto que consta de la redacción de las acciones que definan la actividad, el lugar, día, hora de la conferencia del investigador (Cerezuela 2013, $13,14)$.
Para las jornadas académicas se desarrolló una hoja de ruta que sea una especie de guía metodológica para la intervención del investigador. La hoja de ruta identificó la página de transmisión en vivo vía streaming. Datos como la fecha de inicio y finalización de las jornadas académicas, las instrucciones con el día y fecha de la participación del conferencista, los documentos que deben ser enviados para la elaboración del arte y organizar las diapositivas o fotografías de la ponencia y finalmente la agenda con el orden de las actividades: bienvenida, presentación, conversatorio, preguntas y saludos del público.

\section{Hoja de ruta}

\begin{tabular}{|c|c|}
\hline \multicolumn{2}{|c|}{ "Jornadas Académicas de Historia por el Bicentenario de Ambato" } \\
\hline Datos: & $\begin{array}{l}\text { Página de Facebook "Ambato ayer y hoy" (https://www.facebook.com/ } \\
\text { AmbatoAyeryHoy) } \\
\text { Página alternativa de Facebook "Quito ayer y hoy" (https://www.facebook.com/ } \\
\text { QuitoAyeryHoy) }\end{array}$ \\
\hline Fecha de inicio: & $20-08-2020$ \\
\hline Fecha de finalización: & $05-11-2020$ \\
\hline Instrucciones: & $\begin{array}{l}\text { Su participación se realizará el día miércoles a las } 15 \text { h00 hasta las } 16 \mathrm{~h} 00 \text {, le } \\
\text { solicitamos conectarse unos } 15 \text { minutos antes para probar audio y video. } \\
\text { La transmisión se realizará mediante la plataforma zoom. Se puede hacer uso de } \\
\text { diapositivas o fotografías, tendrá la opción de compartir habilitada. } \\
\text { El link de la transmisión en vivo se enviará una vez empezado el programa al correo } \\
\text { y a WhatsApp para que pueda compartirlo a sus contactos y puedan seguir la } \\
\text { transmisión en vivo. }\end{array}$ \\
\hline Documentos & $\begin{array}{l}\text { Nombres y apellidos completos } \\
\text { Breve hoja de vida de } 8 \text { a } 10 \text { líneas } \\
\text { Fotografía } \\
\text { Tema }\end{array}$ \\
\hline
\end{tabular}




\begin{tabular}{|l|l|}
\hline Agenda: & Bienvenida \\
& Presentación \\
& Conversatorio \\
& Preguntas y saludos del público \\
\hline
\end{tabular}

Fuente: Página de Facebook "Ambato ayer y hoy"

Elaborado por: Elaborado por: Jéssica Pamela Torres Lescano, Rómulo Arteño Ramos, Hernán Paul Ortiz Coloma, Mery Elizabeth Zabala Machado

Queremos detenernos un momento para profundizar las ventajas de la tecnología streaming como una estrategia didáctica en sí para fomentar la interacción en entornos virtuales de aprendizaje. Las múltiples posibilidades de incluir laminas, fotografías, videos y los foros interactivos son amplias. Por eso, en las Jornadas académicas se deja la posibilidad de que el conferencista seleccione la mejor estrategia didáctica para explicar su contenido.
La estrategia más utilizada en las Jornadas académicas fue la inclusión de presentaciones en power point. En las láminas se proyectaron textos enormes que podrían aburrir a los espectadores, por el contrario se eligieron fotografías de las fuentes primarias que fueron usadas para las investigaciones, imágenes del trabajo de campo, cuadros estadísticos y cartografía elaborada por los autores.

Imagen 3

\section{Ponencia "Tiempo de Fiesta: Celebraciones Cívicas en Ambato del siglo XX"}

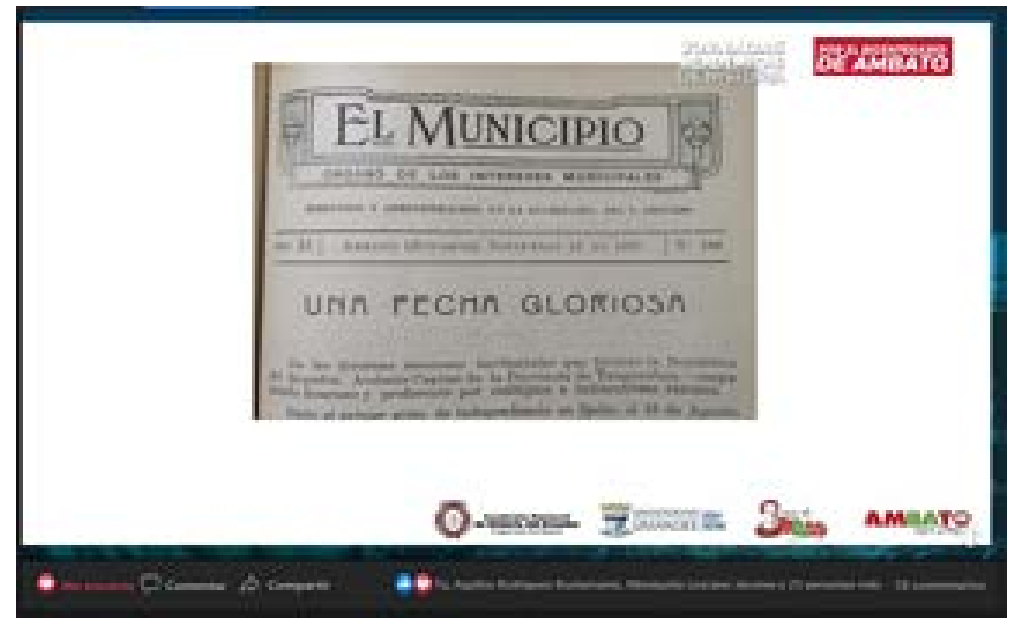

Fuente: Página de Facebook "Ambato ayer y hoy" https://www.facebook.com/Ambatoayeryhoy/ videos/1580983635415056/

Elaborado por: Jéssica Pamela Torres Lescano

REVISTA B OLETIN REDIPE 10 ( 9 ) : $3558-370$ - SEPTIEM BRE $2021-1$ S S N $2256-1536$ 
De forma que, el formato de presentación y desarrollo de las actividades formativas que se incluyen en un aula virtual tiene que responder a realidades específicas que la tecnología pueda aportar de modo particular a los procesos educativos (simulaciones, visualización de procesos, etc.) (Barbera y Badia 2005, 19). La selección de las herramientas para socializar la investigación no es casual y debe elaborarse con tiempo previo para mejorar su efectividad.

\section{ANÁLISIS E INTERPRETACIÓN}

Lo que proponemos aquí es orientar la reflexión alrededor de dos aspectos. El primero sobre las iniciativas de conmemoraciones cívicas desde el sector académico privado, que bien pueden ser alternativas para releer acontecimientos históricos integrando instituciones e investigadores. $\mathrm{Y}$ el segundo, reflexionar sobre las ventajas de la estrategia de educación y socialización del streaming en la divulgación científica a públicos más amplios. Como se mencionó, al inicio, se rastrearon estos dos factores en el caso de la investigación "Tiempo de Fiesta: Celebraciones cívicas del siglo XX" en las Jornadas Académicas de Historia por el Bicentenario de Ambato

Desde esta perspectiva, parte de pensar el momento independentista como un proceso también significa releer las fiestas cívicas. En la revisión de las celebraciones se considerarán: el Concejo Cantonal y las propuestas de los sectores artesanales para el festejo del 12 de noviembre de cada año en Ambato. Es decir, las actividades de la institución local oficial y las iniciativas de los sectores populares.

El Concejo Cantonal se miraba a sí mismo con la labor patriótica de organizar la celebración de la emancipación política de Ambato y de Tungurahua. Las actas del Concejo Cantonal mencionan "que el municipio está obligado a conmemorar dignamente los acontecimientos que han dado renombre al pueblo que representa" (Actas del Concejo Cantonal 1927). No se trató de una celebración improvisada sino pensada con anterioridad. La planificación empezaba en los meses de septiembre, octubre y algunas ocasiones desde el inicio de la gestión del Concejo Cantonal. El aniversario de emancipación se aprovechó para inaugurar obras municipales o presentar el informe de las labores municipales anuales. La comisión de organización tenía amplias facultades para llevar a cabo las gestiones preparatorias. El proyecto del programa de festejos era socializado en el seno del Concejo Cantonal para ser modificado o aprobado. El presupuesto para la celebración cívica variaba entre la suma de 2000 sucres hasta 3000 sucres proveniente de los fondos extraordinarios e imprevistos de la Ordenanza Presupuestaria.

En el siglo XX fue frecuente que el Concejo Cantonal incorpore actos de beneficencia en el programa de festejo. El premio anual 12 de noviembre fue la iniciativa de los miembros de la entidad cantonal que sugería destinar anualmente a una familia de escasos recursos económicos la cantidad aproximada de 1000 sucres. Entre los requisitos para participar en el sorteo estaban: "tener a su legítimo y exclusivo cargo una familia compuesta por lo menos de cuatro miembros, en su totalidad. Que sea, dicha familia, oriunda o nativa, siquiera en su mayor parte de este cantón, y en todo caso, que tenga residencia fija en él. Carecer de renta propia, segura y suficiente de casa de habitación y de parientes o deudos cercanos y pudientes a cuyos bienes de fortuna pudiesen los interesados tener derecho por causas de fallecimiento o donación de aquellos. Vivir de su empleo o de sus convivientes, su industria u oficios honradamente. Tener buenos antecedentes, gozar de buena opinión y observar y procurar que observen todos los suyos, una conducta y moralidad intachables" (Actas del Concejo Cantonal 1927). Un año más tarde y con la voluntad de beneficiar a mayor 
número de personas la cantidad se dividió en cinco premios de doscientos sucres cada uno.

La fecha cívica fue vista como la oportunidad para agasajar el buen desempeño de los trabadores locales. En palabras de los integrantes del Concejo Cantonal su labor se encaminó a "contribuir con actos filantrópicos y reales al bienestar de los asociados. Que se contiene también entre los deberes primordiales de la Corporación, el de estimular, por este u otros medios análogos, la paciente laboriosidad y honradez y galardonar de algún modo la abnegación, el espíritu de sacrificio y demás virtudes domésticas de los mismos" (Actas del Concejo Cantonal 1927). No tardó en proponerse el premio 12 de noviembre para el empleado municipal en el año 1928. La honorabilidad, la antigüedad y el buen desempeño eran las características del ganador. Igualmente, el premio Cuesta Garcés, llamado así por el jurisconsulto Ignacio Cuesta Garcés, premiaba a la empleada que se distinga por sus méritos. Funcionarias de la Oficina Telefónica y la Clínica Tarquino Toro Navas fueron las ganadoras en la primera edición (Actas del Concejo Cantonal 1928)

Los sectores artesanales se sumaban a los festejos de la fecha cívica con iniciativas emprendidas con la cooperación del Concejo Cantonal. El Comité de Zapateros "12 de noviembre" propuso la organización de una Feria de muestras. El Liceo Cevallos fue el espacio que presenció durante varios años la exhibición de calzado convocada por los productores locales. Las premiaciones incluían una tarjeta de oro para el comité organizador, una medalla de oro para el mejor expositor y menciones honoríficas para los participantes. A decir del Concejo Cantonal: "las iniciativas de los obreros deben ser atendidas en apoyo y galardón para sus esfuerzos" (Acta del Concejo Cantonal 1929). Como se pudo ver, el 12 de noviembre en el siglo XX motivó el esfuerzo de varias secciones de la sociedad local para su celebración. Los festejos incluyeron actividades académicas, así como actos de beneficencia y exposiciones de los sectores obreros locales.

En el 2020, nos insertamos en ese marco de celebraciones bicentenarias en un contexto de pandemia. Esta ocasión se necesitan alianzas entre instituciones, modificar las plataformas de interacción e incluir nuevas voces que nos ofrezcan nuevas miradas sobre la celebración del bicentenario. Las Jornadas Académicas de Historia son un ejemplo efectivo de la divulgación científica desde sectores privados que se encuentran preocupados por la educación comunitaria.

\section{Imagen 4: Evaluación de resultados}

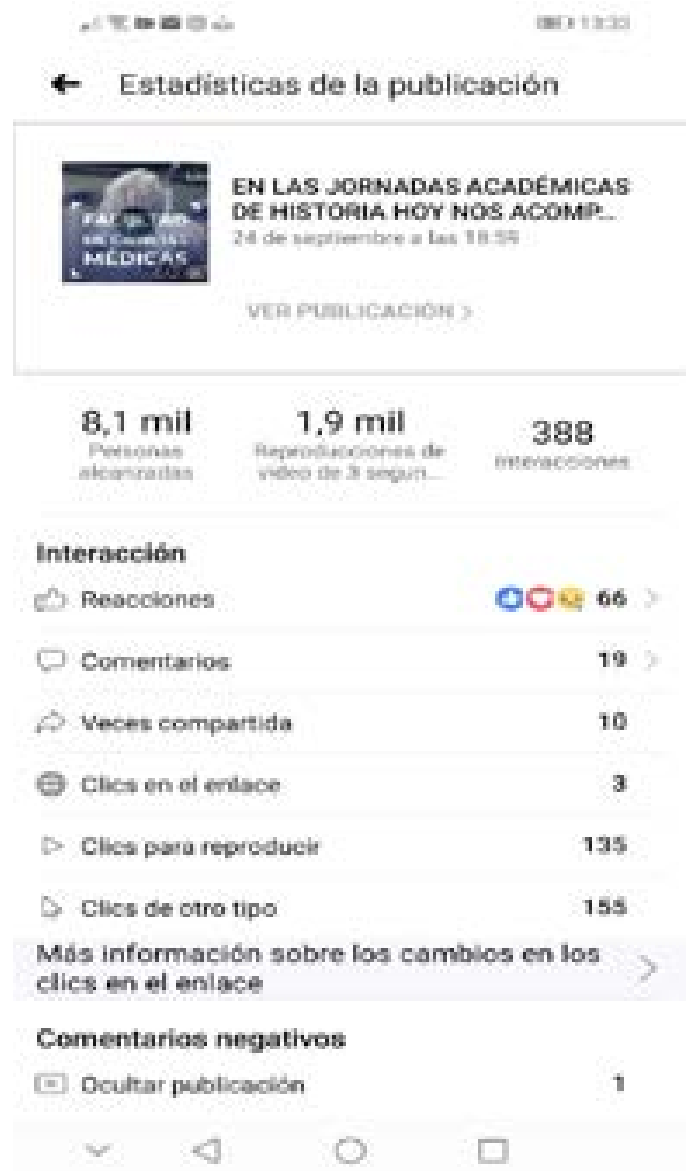

Fuente: Página de Facebook "Ambato ayer y hoy"

Elaborado por: Jéssica Pamela Torres Lescano 
Las estadísticas que la página de Facebook "Ambato ayer y hoy" registra es de 8100 personas alcanzadas y 1900 reproducciones de video de 3 segundos. La totalidad de interacciones es de 388 incluyendo reacciones, comentarios. Las veces que fue compartida la publicación tiene un total de 10. En un entorno virtual, el moderador y el investigador deben gestionar la interacción con el público. Se debe considerar que en un evento a distancia la interacción depende de conexiones, velocidad de transmisión de datos, video y audio de calidad (Castillo 2020, 346).

\section{CONCLUSIONES}

No han sido pocas las reflexiones emprendidas desde la Academia con motivo de la celebración del Bicentenario de Independencia en los países de América Latina. Preguntas como ¿qué conmemorar en el bicentenario? y, ¿por qué es importante? fueron frecuentes en los seminarios, congresos y talleres de Historia desarrollados este 2020. Las revoluciones hispanoamericanas siguen siendo motivo de estudio después de 200 años. $Y$ es que, la mirada al pasado de cómo analizar la Independencia se ha transformado significativamente. La historiografía tradicional narró la independencia como un momento encaminado hacia la construcción de los estados nación. La elaboración de estas narrativas fundacionales se acompañó de la exaltación de los héroes (en su mayoría hombres) y las grandes batallas. Es decir, los historiadores del siglo XIX se distinguieron por elaborar relatos con una clara expresión épica. De esta manera, la Independencia se instauró en el relato nacional como la ruptura total con el Antiguo Régimen.

Estudiar las conmemoraciones cívicas es acercarse al imaginario de la ciudad de Ambato del siglo XX: la construcción de la nación desde la Patria Chica. Es decir, la agencia de los márgenes en la formación de los Estados y su organización para festejar el aniversario de la independencia, gloriosa efeméride, fecha clásica, fecha gloriosa o fiesta cívica (como se denomina en las fuentes). Pensar el bicentenario hoy es poner en cuestionamiento no sólo una sino varias aristas: es repensar el papel de las periferias y los espacios urbanos en los festejos (Idrovo 2009, 136), es lograr organizar una verdadera celebración, una fiesta sin exclusiones (Borrero 2009, 141), es organizar una verdadera celebración, una fiesta sin exclusiones (Landázuri 2009, 139). A estas propuestas sumaría: planificar un bicentenario sin anacronismos, sin usos políticos de la historia.

En este contexto, la educación via streaming es una alternativa válida para la socialización de eventos culturales. Entre las ventajas de la estrategia virtual están la oportunidad de interactar en tiempo real y la posibilidad de realizar preguntas, comentarios con el investigador. La plataforma streaming posibilita romper las barreras de la distancia y de tecnología (121). Consideramos que streaming tiene múltiples posibilidades que aún restan ser exploradas. Por su doble característica sincrónica y asincrónica se convierte en una oportunidad de formar un archivo digital de conferencias que se perpetúe en el tiempo.

\section{FUENTES PRIMARIAS:}

\section{Archivo Nacional Seccional Tungurahua}

Actas del Concejo Cantonal 1927

Actas del Concejo Cantonal 1928

Actas del Concejo Cantonal 1929 


\section{REFERENCIAS BIBLIOGRÁFICAS}

Ander Egg, Ezequiel y María José Aguilar Idáñez. 2005. Cómo elaborar un proyecto: guía para diseñar proyectos sociales y culturales. Instituto de Ciencias Sociales Aplicadas ICSA. https://www.researchgate.net/profile/Cesar-Munder-Calderin/publication/320263416_COMO_ ELABORAR_UN_PROYECTO_GUIA_PARA_DISENAR_PROYECTOS_SOCIALES_Y_ CULTURALES/links/59d83f62aca272e6095f96a2/COMO-ELABORAR-UN-PROYECTOGUIA-PARA-DISENAR-PROYECTOS-SOCIALES-Y-CULTURALES.pdf

Barbera, Elena y Antoni Badia. 2005. Hacia el aula virtual: actividades de enseñanza y aprendizaje en la red. Revista Iberoamericana de educación, 36(9), 1-21. https://d1wqtxts1xzle7. cloudfront.net/44701676/AulasVirtuales.pdf?1460567216=\&response-content-dispo sition=inline\%3B+filename\%3DHACIA_EL_AULA_VIRTUAL_ACTIVIDADES_DE_ ENS.pdf\&Expires $=1618675796 \&$ Signature $=$ L7 $-X Z a S R 6 U 9 d b h 4 i 4$ ts 4926sqUpJzk1 ypHI8tqDVtZBeFmCllbIB-Fuqeg1 RqFI25z7bfj8oMiCCqgS-T3OVTPf0G2cFQO2TVdlcIzW kBn34B89NhERRspfqb9DDUECfXuF8gL gaCKW--NiH-SsfOlq473QNUzp CRSry a9sqthJP2vv zCs0mDFpbF- 7yVM 78ErB QLJ724YsdgMliE95q1iLa1Ocnjvmasld2VPOkn1wHtll87x5mRIPJfzX9ZqtcW8ss-v0xaxWKiusYf6CuYkpeUBLGYUpjhNLIhqF4zc3-INdX KedIDvt9bRUGRwdl8s52k1poDw_\&Key-Pair-Id=APKAJLOHF5GGSLRBV4ZA

Cáceres-Piñaloza, Katherine. 2020. Educación virtual: Creando espacios afectivos, de convivencia y aprendizaje en tiempos de COVID-19. CienciAmérica, 9(2), 38-44. http://cienciamerica.uti.edu. ec/openjournal/index.php/uti/article/view/284/424

Cerezuela, David. 2013. 7.1 Diseño integral de proyectos culturales. Observatorio Cultural del Proyecto Atalaya. http://atalayagestioncultural.es/pdf/07.1.pdf

Eidus - Concello da Coruña. 2020. Plan de comunicación. Fondo Europeo de Desarrollo FEDER. https:// www.coruna.gal/descarga/1453644346910/PlanDeComunicacionEidusCoruna.pdf

Hidrovo Quiñónez, Tatiana María del Carmen, Carlos Landázuri Camacho, Ana Luz Borrero Vega, Enrique Ayala Mora y Ángel Emilio Hidalgo Ortiz. ¿Cómo conmemorar la independencia?”. Procesos: revista ecuatoriana de historia. 29 (I Semestre, 2009): 133-146.

Kotler, Philip. 2001. Dirección de mercadotecnia: análisis, planeación, implementación y control. Magíster en Administración-Tiempo Parcial 29, ESAN. https://anafuenmayorsite.files.wordpress. com/2017/08/libro-kotler.pdf

Lancheros-González, Magnolia Jordan, Herrera-Marchena, Pilar Morantes-Hernández, Ricardo Buitrago-Bernal, Iván López-Buitrago, Jorge Pombo. 2021. Un espacio virtual para vivir la ciencia y la tecnología por medio de streaming. Revista Internacional de Pedagogía e Innovación Educativa, 1(2), 103-124. https://editic.net/ripie/index.php/ripie/article/view/44/35

Nicola, Gerardo. 2019. Ambatiqum: Antología biográfica tungurahuense. Megagraf Imprenta.

Tur-Viñes, Victoria y Juan Monserrat-Gauchi. 2014. El plan estratégico de comunicación. Estructura y funciones. Razón y palabra, 18(88). https://www.redalyc.org/pdf/1995/199532731046.pdf 